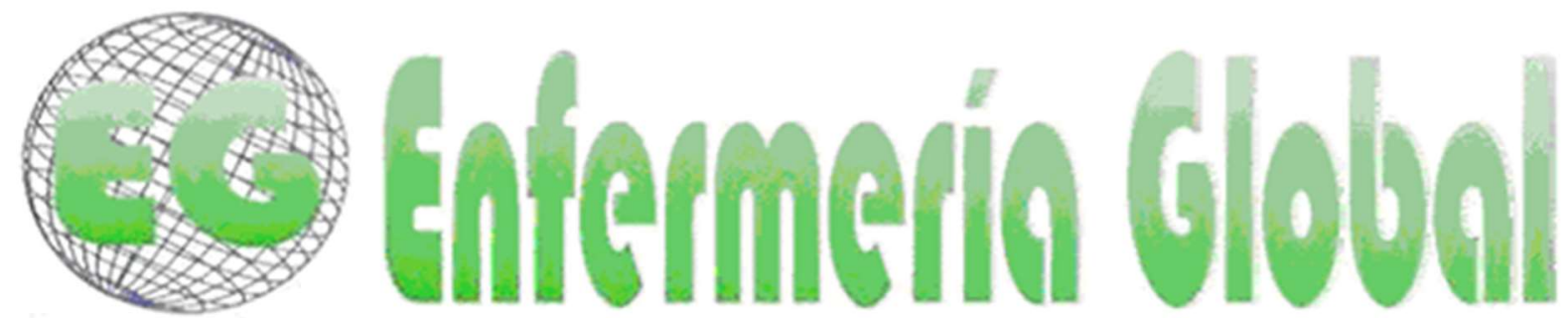

\title{
ORIGINALES
}

\section{Realidades de la práctica de la automedicación en estudiantes de la Universidad del Magdalena}

Realities of the practice of self-medication in students of the Magdalena University

\author{
Haidy Oviedo Córdoba ${ }^{1}$ \\ Carolina Cortina Navarro ${ }^{2}$ \\ Javier Andrés Osorio Coronel ${ }^{3}$ \\ Sandra Marcela Romero Torres ${ }^{3}$
}

${ }^{1}$ Enfermera, Doctoranda de Enfermería Universidad de Carabobo. Miembro del Grupo de Investigación Gicce. Docente de planta Programa de Enfermería, Facultad de Ciencias de la Salud, Universidad del Magdalena. Colombia. hoviedo@unimagdalena.edu.co

2 Enfermera, Especialista en Gerencia y Auditoría de la calidad en salud. PhD en Ciencias de la Educación. Docente Facultad de Ciencias de la Salud. Miembro del Grupo de Investigación Gicce. Universidad del Magdalena Santa Marta Colombia

${ }^{3}$ Semillerista. Grupo de investigación Gicce. Programa de Enfermería Facultad de Ciencias de la Salud. Universidad del Magdalena. Colombia.

\section{https://doi.org/10.6018/eglobal.430191}

Recibido: $31 / 05 / 2020$

Aceptado: $21 / 12 / 2020$

\section{RESUMEN:}

La automedicación forma parte del autocuidado y es considerada como un recurso de salud pública primaria en el sistema de atención de la salud.

Objetivo: Determinar la prevalencia y patrones de consumo que influyen en la automedicación de los estudiantes de la Universidad del Magdalena

Métodos: El estudio corresponde a una investigación de carácter descriptivo, de corte transversal, y enfoque cuantitativo; la muestra se determinó por conglomerados, conformada por 312 estudiantes activos matriculados en pregrado de la Universidad del Magdalena de la ciudad de Santa Marta. Resultados: La práctica de la automedicación se vio reflejado en un $97 \%$; los motivos por los cuales se automedican los estudiantes tienen relación con la aparición de síntomas tales como el dolor y síntomas gripales, los cuales son tratados a partir del consumo de medicamentos tipo analgésicos, antiinflamatorios, con una prevalencia del $84,26 \%$. El principal motivo para automedicarse se relaciona con la levedad de los sintomas; el consejo de familiares en un $46,87 \%$ refleja su fuente de información; existe influencia por la publicidad especialmente televisión e internet; finalmente el $71 \%$ tiene conocimiento de las consecuencias que acarrea el automedicarse.

Conclusiones: La práctica de la automedicación en la población universitaria es alta; los factores influyentes se relacionan con aparición de sintomas, el consejo de familiares, la influencia de la publicidad, la levedad de los sintomas y la falta de tiempo para visitar al médico.

Palabras claves: Automedicacion, Autocuidado, medicamentos 


\begin{abstract}
:
Self-medication is part $f$ self-care and is considered as a primary public health resource in the health care system.

Objective: To determine the prevalence and consumption patterns that influence the automation of the students of the Universidad del Magdalena.

Methods: The study corresponds to a descriptive cross-sectional and quantitative approach investigation; the sample was determined by conglomerates, made up of 312 active students enrolled in undergraduate studies at the Universidad del Magdalena the city of the Santa Marta.

Results: The practice of self-medication was reflected in $97 \%$; the reasons for which the students selfmedicate are related to the appearance of symptoms stories, such as pain and flu-like symptoms, which are treated from the consumption at analgesic, anti-inflammatory drugs with prevalence of $84,26 \%$. The main reason for self-medication is related to the mildness of the symptoms; the council of relatives in $46,87 \%$ reflects their source of information; there is influence by advertising especially television and internet; finally $71 \%$ are aware of the consequences of self -medication.

Conclusions: The practice of self-medication in the University population is high; influencing factors are related to the appearance of symptoms the advice of relatives, the influence of advertising the mildness of symptoms and lack of the time to visit the doctor.
\end{abstract}

Key words: Self-medication, Self -care, medications

\title{
INTRODUCCIÓN
}

A través de la historia del cuidado humano, pueden identificarse figuras que fueron destacándose dentro de las comunidades primitivas siendo expertos 0 bien acumulando la habilidad en el mantenimiento y conservación de la vida y la salud ${ }^{1}$, por ello a estos miembros dentro de las etapas de la evolución del cuidado se les reconoce el dominio de las plantas y la generación de los primeros fármacos para la curación de enfermedades.

En la medida que fue evolucionando el concepto de salud, enfermedad y las figuras del cuidado, fueron dándose también cambios en la farmacopea, unido esto al desarrollo de la ciencia, hasta desarrollarse totalmente como un componente importante en el manejo terapéutico de las situaciones de salud.

La automedicación aparece como uno de los fenómenos para enfrentar socialmente la enfermedad, siendo definida como la administración por decisión propia, o por consejo no cualificado, de medicamentos ya sea para aliviar un síntoma o curar una enfermedad, o simplemente como la libre decisión de tomar medicamentos sin prescripción médica ${ }^{(2)}$

Según la Organización Mundial de la Salud (OMS), la automedicación se define como la selección y uso de medicamentos por los individuos para tratar enfermedades o síntomas auto reconocidos. La automedicación forma parte del autocuidado y es considerada como un recurso de salud pública primaria en el sistema de atención de la salud ${ }^{(3)}$.

Sin embargo, de manera alarmante la automedicación ha ido en aumento como una acción voluntaria que implica riesgos para la salud, no solo por los efectos que puede en algún momento suscitarse por el uso indiscriminado del medicamento, sino también el enmascarar síntomas de alguna enfermedad, la prolongación de la misma, la resistencia o llegar a agravar la misma enfermedad incluso en grupos más vulnerables como niños, mujeres embarazadas y lactantes. 
Es un hecho que este fenómeno se ve afectado por los determinantes políticos, económicos, sociales y culturales que caracterizan el entorno dándose la facilidad de compra y el uso de medicamentos sin control, lo que puede traer consecuencias a corto mediano y largo plazo como la aparición de reacciones adversas, complicaciones en el diagnóstico oportuno y la drogo resistencia (3).

Por otra parte, organizaciones como la Federación Farmacéutica Internacional y la industria mundial de la automedicación responsable, señalan algunos aspectos positivos de esta práctica de automedicación, entre los que se encuentran la menor demanda sanitaria a dolencias menores que no la requieren, con lo cual reducen costos al sistema sanitario, la mayor accesibilidad del paciente al medicamento lo que le lleva a un alivio más pronto de su dolencia y la implementación del cuidado de su salud enmarcando la automedicación como uno de los autocuidados que la persona asume dentro de su estilo de vida, nutrición e higiene.(4).

Diversos autores reconocen al abordar este tema, que dicha práctica tiene tanto beneficios como riesgos, para ello es de vital importancia que el paciente sepa distinguir las situaciones en las que se requiere que sea un profesional de la salud quien resuelva la condición de salud o bien pueda a través de su propio conocimiento solventarlas; sin embargo son diversos los factores que pueden interferir en esta decisión, tales como el ahorro de tiempo y dinero al momento de solicitar una consulta médica, más aun si el sistema de salud tiende a colapsarse ${ }^{(5)}$, o bien el disminuir la pérdida de tiempo laboral o los desplazamientos entre otros; aunque pueden también revisarse las desventajas que esta práctica puede acarrear para las personas, siendo el más representativo los riesgos para la salud que se presentan por la aparición de reacciones adversas, interacciones medicamentosas, aumento de la resistencia bacteriana, aumento de la duración de la enfermedad, por nombrar solo algunos ${ }^{(6)}$.

En Latinoamérica estudios realizados en Ecuador (7), Perú (8), Argentina (9) y México(10), han reflejado la prevalencia de esta práctica en diferentes ambientes y se ha logrado evidenciar la fuerte influencia que tiene en el ámbito universitario, siendo significativa la práctica de la automedicación en estudiantes que cursan carreras del área de la salud, a partir de semestres en los cuales ya han visto el curso de farmacología.

Estudios similares realizados en la Universidad del Rosario en Colombia indican que la mayor frecuencia de automedicación la obtienen los estudiantes de la facultad de salud especialmente aquellos que cursan grados clínicos o semestres avanzados (11).

Ahora bien, en lo que respecta a la ciudad de Santa Marta y en especial a la Universidad del Magdalena, no se conocen registros que permitan conocer el comportamiento de este fenómeno, por ello se hace interesante indagar si esta práctica es igual de común dentro de los estudiantes de la universidad, toda vez que nuestro sistema de salud permite la venta libre de medicamentos.

\section{MATERIALES Y METODOS}

El estudio corresponde a una investigación de carácter descriptivo de corte transversal, y enfoque cuantitativo. El universo para la realización de este estudio lo conforman los estudiantes de pregrado de la Universidad del Magdalena matriculados en el II periodo del año 2019; se utilizó la fórmula para poblaciones finitas para 
estudios descriptivos a fin de obtener el tamaño de la muestra; el cálculo de tamaño de muestra fue de 300 estudiantes, y se realizaron 330 encuestas contemplando un porcentaje de pérdidas del $8 \%$. De las 330 encuestas se analizaron 305, ya que las excluidas no fueron diligenciadas en su totalidad, o pertenecían a un programa técnico de la universidad.

El muestreo fue por conglomerado de las diferentes áreas que componen la Universidad, mediante la selección de números aleatorios en Excel de salones y espacios abiertos, posteriormente se hizo el sorteo quedando seleccionados ocho salones y dos áreas comunes. La técnica de recolección de datos se realizó por encuesta directa por parte de los investigadores; para cumplir con los objetivos de la investigación, como instrumento se utilizó un cuestionario de 15 preguntas que valoran las variables propuestas, validado por Flores y Santos en su trabajo sobre la automedicación en estudiantes de las Facultades de Farmacia, Odontología, y Enfermería en la UNAP Perú (12); el cuestionario se divide en tres partes; la primera permitirá realizar la caracterización de la población de estudio por ello estarán los datos socio económicos y demográficos; la segunda está enfocada hacia el patrón de automedicación en la cual se busca recolectar información relacionada con tipo de medicamentos consumidos, motivos para automedicarse, los referentes informativos y los lugares de adquisición que favorecen la conducta y finalmente la tercera parte indaga la percepción del encuestado respecto a la automedicación.

Se utilizaron técnicas de estadística descriptiva para la presentación de las variables de acuerdo con su naturaleza y escala de medición que sirvieron para explicar el comportamiento de la automedicación en la población estudio. Se creó la base de datos en EXCEL, se realizó doble digitación y validación de la información, posteriormente, la base de datos se exportó a EPI INFO VERSION 7.2.3.1 para el análisis. Se calcularon medidas de frecuencia absoluta y relativa y medidas de tendencia central para las variables cuantitativas según su distribución y proporciones.

\section{Aspectos éticos}

El restudio fue aprobado por el Comité de ética de la Facultad de Ciencias de la Salud de la Universidad del Magdalena. Siguiendo las consideraciones éticas la investigación en seres humanos contemplada en la declaración de Helsinki ${ }^{(13)}$ y de la Resolución 008430 de 1993 del Ministerio de Salud (14), esta investigación se considera de bajo riesgo y con el fin de proteger los derechos de los participantes, se les presentó de manera individual el proyecto, además se les informó de que los resultados de la investigación solo se utilizaran con fines académicos, se diligenció el formato de consentimiento informado siendo así su participación completamente voluntaria y garantizando los principios de confidencialidad y privacidad.

\section{RESULTADOS}

Se encontró que el 97\% emplearon medicamentos por cuenta propia, sin prescripción médica, siendo el sexo masculino con mayor porcentaje $56 \%$. El rango de edad donde se observó mayor practica de automedicación es entre $16-19$ años y los dos primeros años de estudio de las facultades de Ingeniería y Salud. 


\section{Grafica 1. Distribución por programa de los estudiantes de la Universidad del Magdalena encuestados}

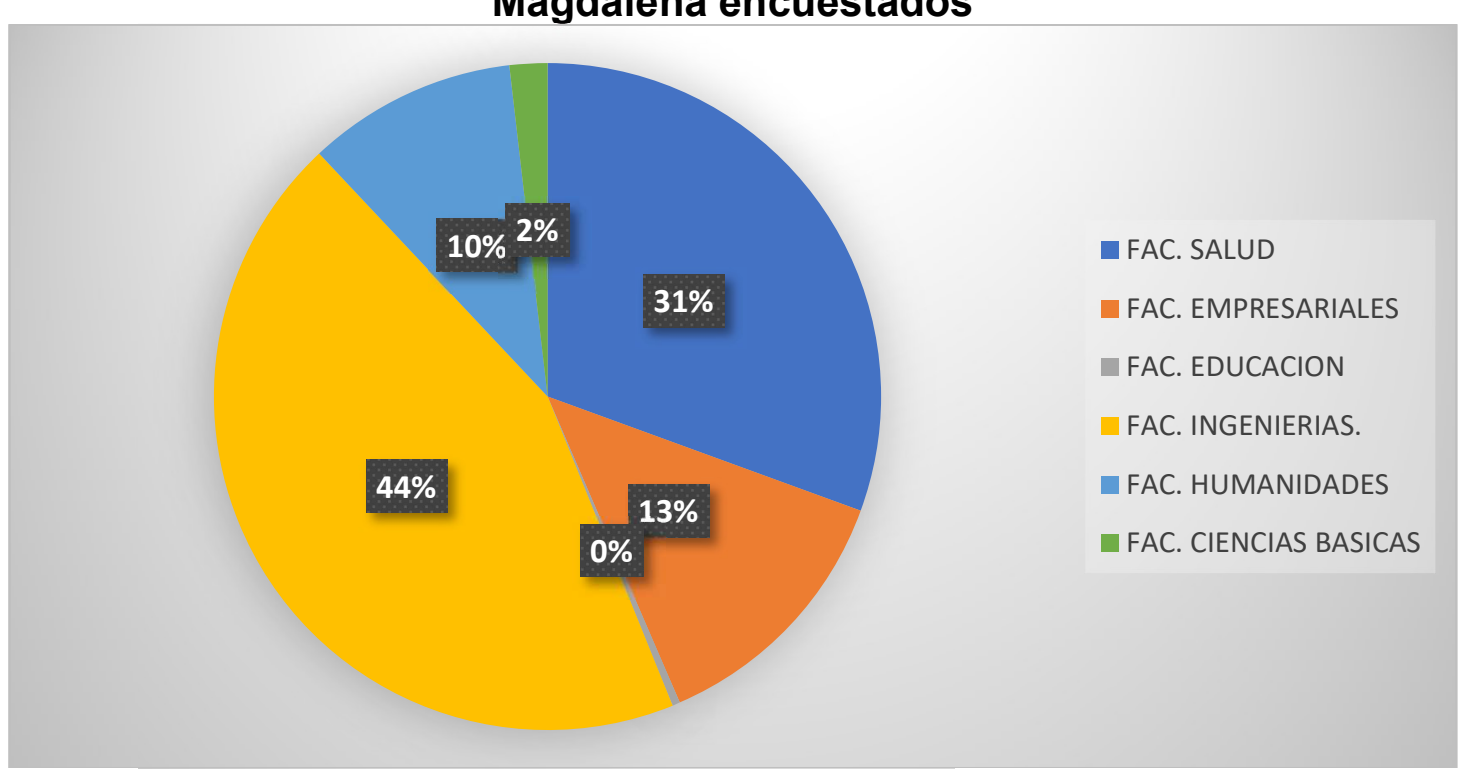

Los estudiantes que se auto medican manifestaron que los principales síntomas o signos por los cuales realizaron esta práctica en algún momento fueron dolores \{cabeza, menstrual, estomacal, garganta, etc.\} $(87,87 \%)$, síntomas gripales \{resfrío, fiebre, tos\} $(86,56 \%)$, por otro lado, de las mujeres que refirieron auto medicarse, el $23,13 \%$ indicó que alguna vez lo ha hecho para la prevención del embarazo.

\section{Grafica 2. Síntomas o signos que motivaron la automedicación de los estudiantes de la Universidad del Magdalena 2019}

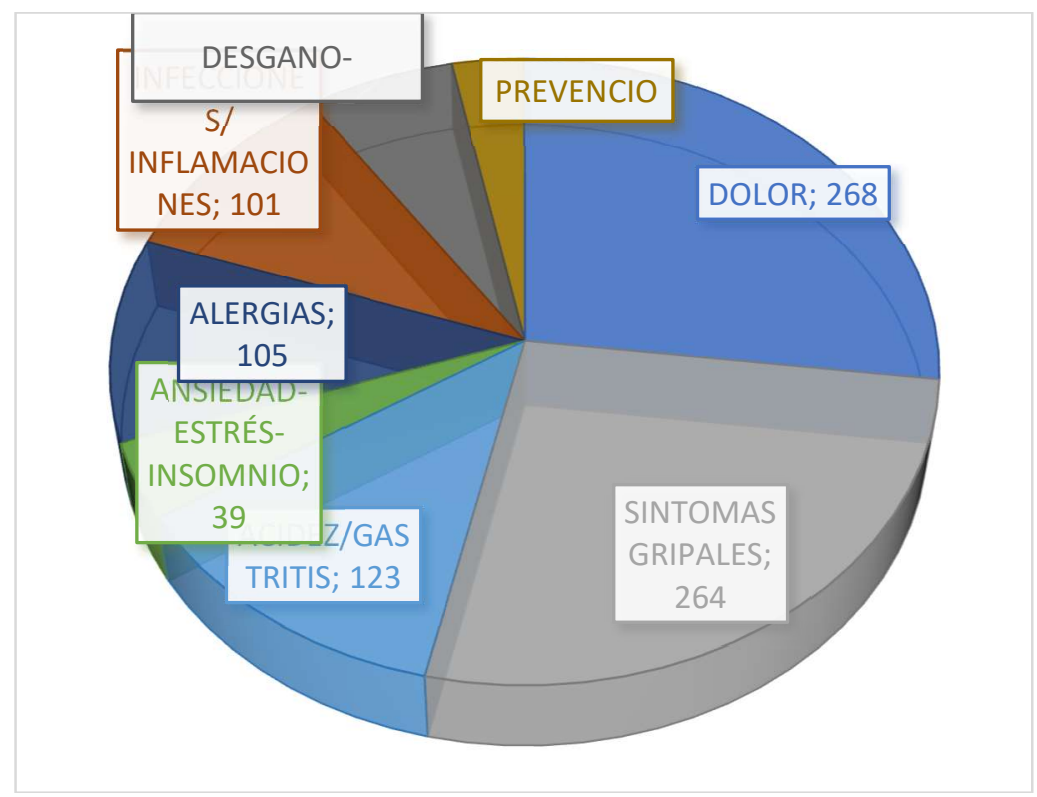

En relación con los motivos de consumo de medicamentos sin prescripción por lo cual los estudiantes deciden realizar esta práctica de automedicación, se encuentra que la principal causa es debida a la levedad de los síntomas en un $74,75 \%$ seguido por el fácil acceso y comodidad en un $50,49 \%$, continuado por el no gusto a ir al médico en un $34,10 \%$. 


\section{Grafica 3. Relación y tipo de medicamentos más utilizados por los estudiantes de la Universidad del Magdalena 2019 II}

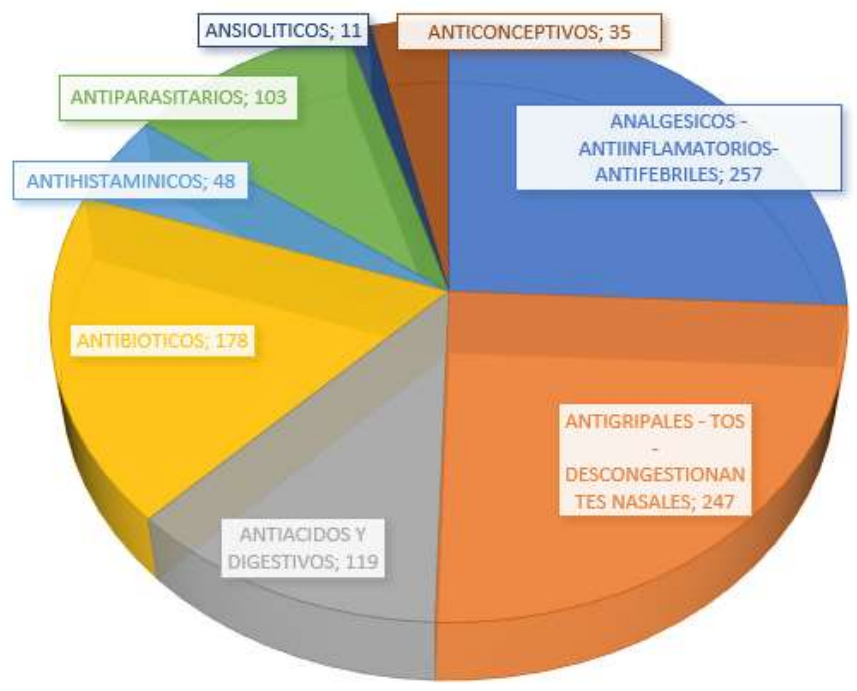

En relación al tipo de medicamento utilizado en la automedicación, se encontró que los principales grupos de medicamentos que han utilizado son los analgésicos, antiinflamatorios, antipiréticos con una prevalencia del $84,26 \%$ (257 alumnos lo utilizaron) y los antigripales, antitusivos, y los descongestionantes nasales con un $80,98 \%$ de prevalencia (247 estudiantes lo consumieron) seguidos por los antibióticos con un $58,36 \%$.

Grafica 4. Porcentaje de las Fuentes donde los estudiantes de la Universidad del Magdalena adquieren información sobre medicamentos.

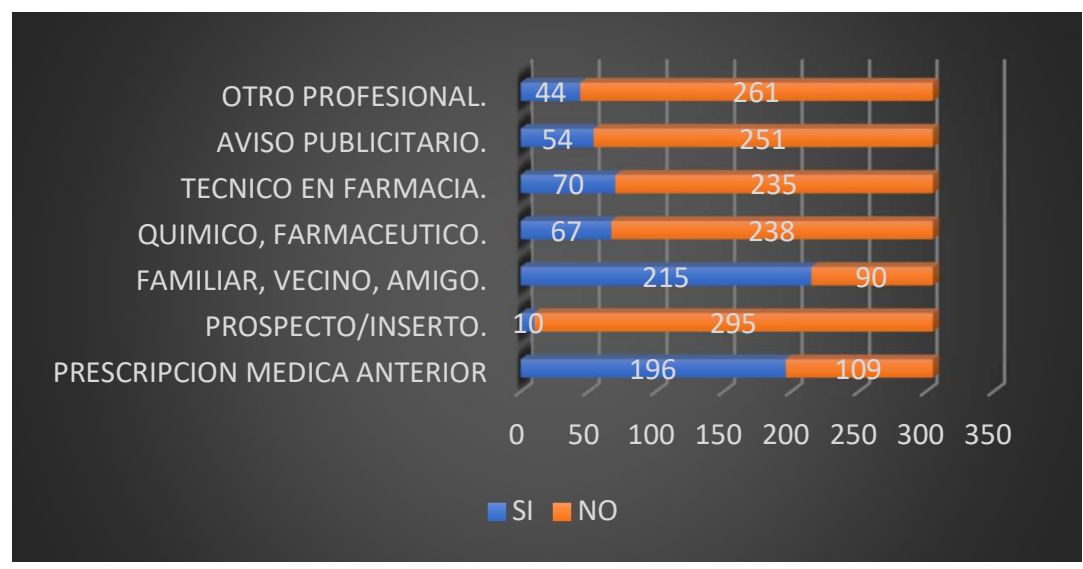

Otro de los aspectos que se indagaron en la población de estudiantes encuestados, corresponde como lo muestra la gráfica 4 a las principales fuentes de información que utilizan los estudiantes para decidir tomar algún medicamento sin la prescripción de un médico; los resultados reflejan el consejo de un familiar, un vecino o algún amigo $(70,49 \%)$ a lo que le sigue alguna prescripción médica anterior $(64,26 \%)$, seguido por la opinión de un técnico en farmacia $(22,95 \%)$. 
En relación con la manera como los medios de comunicación o publicidad pueden incidir en la decisión de los estudiantes universitarios en la automedicación; ante la pregunta el 46,89\% refirió que alguna vez consumió medicamentos influenciado por este tipo de difusión, así mismo los principales tipos de publicidad que influyen en esta acción son la televisión en un $43,61 \%$ seguido por el internet con el $21,64 \%$ la radio con el $17,05 \%$.

Por otra parte, las respuestas que los encuestados dieron respecto a la frecuencia con que van al médico reflejan que el $67,21 \%$ de los encuestados asisten a consulta al médico solo cuando está enfermo, el $14,75 \%$ acude al profesional de la salud anualmente, mientras que el $10,16 \%$ lo hace por control de enfermedad y solo el 7,87 va a cita médica mensual.

Durante el desarrollo de la encuesta, el descubrir las razones por las cuales los estudiantes no acuden al médico fue otro de los factores a analizar dentro del fenómeno de la automedicación; una de las razones guarda relación con la ineficiencia de los servicios en un 59,34\%; el $51,48 \%$ de los encuestados no lo hace por la falta de tiempo, el $15,74 \%$ dice que no va por falta de dinero, mientras que la principal razón para el $7,21 \%$ de la población encuestada es la desconfianza en el profesional médico.

En correspondencia, al indagar a la población encuestada sobre el conocimiento de las consecuencias de la automedicación, se encontró que el $71 \%$ de los encuestados refirió conocer los factores que esta práctica pueden desencadenar, mientras que el $29 \%$ no conoce las consecuencias de ingerir medicamentos sin la prescripción de un médico.

\section{DISCUSIÓN}

A partir del análisis de resultados, podemos afirmar que la automedicación es una práctica ampliamente extendida en la población universitaria estudiada alcanzando un 97\%; estas cifras son superiores si comparamos con los resultados de Castronovo C, et al. ${ }^{(5)}$, que en su estudio índican que el 95\% de los encuestados manifestaron auto medicarse alguna vez; así mismo López-Cabra C.A et al.(15) obtuvieron una prevalencia de $79 \%$ en su estudio realizado en la Universidad del Rosario en Bogotá, lo que indica que la automedicación es una práctica cada vez más común entre los jóvenes.

Los resultados disienten con estudios realizados previamente por Guillem (16), en los que aseguran que la tasa de automedicación es más alta en mujeres que en hombres, pero concuerdan con los resultados obtenidos por Moraga C. et al (17), que no observaron diferencias significativas respecto a sexo y edad; puesto que en los resultados del presente trabajo se observa una relación 1:1 siendo la automedicación similar en ambos sexos con tendencia de una ligera inclinación hacia el sexo masculino.

Analizando los resultados en relación con distintas variables los principales motivos que indujeron al consumo de medicamentos fueron los distintos tipos de dolor, síntomas gripales y acidez /gastritis, estas causas concuerdan con el estudio realizado por De Pablo ${ }^{(4)}$ con estudiantes en Caracas donde indicó que los motivos 
por los cuales se indujo la automedicación fueron las cefaleas y las migrañas, los dolores musculares, molestias estomacales. Así mismo se confirma lo revelado por Souza $L$ et al (18), que nos dice que el dolor es factor por el cual más se auto medican los estudiantes. Morillo ${ }^{(19)}$ en su estudio resalta como sintomas la cefalea con un $18 \%$, los cólicos menstruales $16 \%$, la fiebre un $13 \%$ y dolor abdominal $2 \%$.

En cuanto a los grupos de medicamentos más utilizados se encuentra correlación con estudios realizados en países como Brasil (20), Venezuela (21), Paraguay ${ }^{22}$ (22), Chile (23), Perú (24), en los que se refleja que los analgésicos/ antiinflamatorios/ antifebriles son el grupo de medicamentos más utilizados, seguido de los antigripales, antiácidos y antibióticos, así como lo demuestra Morillo ${ }^{(19)}$ en su estudio el uso de analgésicos se reflejó en un $46 \%$, seguido de antigripales $28 \%$ y anticonceptivos $9 \%$, siendo estos últimos diferente a lo encontrado en el presente estudio; así mismo Garrigoza $\mathrm{S}$ et $\mathrm{al}^{(25)}$ en su estudio realizado en Santa Rosa de Araguay en Paraguay evidencia que el $69 \%$ de los medicamentos reportados como de automedicación corresponden a analgésicos, el $11 \%$ a antialérgicos, $6 \%$ antinflamatorios y $3 \%$ antibióticos. A la vez como lo indica Bonilla $Y{ }^{(26)}$, en su estudio realizado en la Universidad Pedro Ruiz Gallo de Lambayeque Perú, demostró que los antigripales $(49,2 \%)$ son el grupo más utilizado por los encuestados, seguidos de antiinflamatorios $(26,9 \%)$, los antibióticos $(21,3 \%)$, los antifebriles $(15,8 \%)$, los analgésicos $(12,5 \%)$. Por el contrario, en el estudio de Naznin Alam, ${ }^{(27)}$ realizado en Bangladesh en el año 2015 se evidencia que los grupos medicamentos que más compraban los estudiantes eran hierbas medicinales y vitaminas y minerales.

Según los datos obtenidos mediante la encuesta, el proceso de automedicación está relacionada directamente con factores importantes dentro del cual se destacan, la levedad de los síntomas en un $74,75 \%$ seguido por el fácil acceso y comodidad en un $50,49 \%$, continuado por la preferencia a no ir al médico en un $34,10 \%$. De igual manera comparando con Tobón $F .{ }^{(28)}$ se puede apreciar que en su estudio los factores que pueden influir en la automedicación son: la deficiencia en la prestación del servicio de salud a gran parte de la población y la dependencia a fármacos, la cultura, la política, la sociedad y la economía; así como el tiempo para acudir a una consulta médica En contraste con. et al, ${ }^{(29)}$ refieren que en su estudio los factores que influyen en la toma de medicamentos son el creer que no necesita una consulta médica, la venta libre de medicamentos y la falta de tiempo.

De manera relevante en este estudio se evidencian otros factores que inciden directamente en la decisión de la automedicación, expresado por los estudiantes encuestados de los cuales destacan las fuentes de información que indujeron a los encuestados a tomar algún medicamento sin la prescripción de un médico, las principales fueron el consejo de un familiar, un vecino o algún amigo $(70,49 \%)$ a lo que le sigue alguna prescripción médica anterior $(64,26 \%)$, seguido por la opinión de un técnico en farmacia (22,95\%); así mismo los medios publicitarios y medios de comunicación influyen a los jóvenes de la Universidad del Magdalena demostrado por el $46,89 \%$ del estudiantado que refirió alguna vez consumir medicamentos persuadido por este tipo de difusión, así mismo los principales tipos de publicidad que influyen en esta acción son la televisión en un $43,61 \%$ seguido por el internet con el $21,64 \%$ la radio con el $17,05 \%$.

Lo anterior coincide con los resultados del estudio realizado en la Facultad de Medicina de la Universidad de San Francisco Xavier de Chuquisaca de Sucre 
(Bolivia)(17) el cual indicó que la automedicación en estudiantes es inducida por el círculo social (amigos y padres), la publicidad de algunos medicamentos, el acceso a internet $(60 \%)$ y la falta de tiempo que induce a los estudiantes de quinto año.

Igualmente, Garrigoza $S$ et al (25), señala que los principales involucrados en este proceso son los familiares y amigos que recomiendan fármacos de acuerdo a su experiencia previa.

Felipe $\mathrm{D}$ et al ${ }^{(30)}$, refiere que el $33 \%$ de los individuos recibieron sugerencia de un familiar, el 7,1\% recibieron sugerencias de un vecino o amigo; mientras que el $42,9 \%$ recibieron sugerencias del personal en la botica y el $16,7 \%$ recibieron sugerencias de otros profesionales de salud no médicos. Además, indica que los principales medios publicitarios que influyen a los encuestados en su estudio fueron la televisión $(56,0 \%)$, paneles publicitarios $(11,9 \%)$ e internet $(25,0 \%)$. Así también Hermoza ${ }^{(31)}$ coincide al reconocer en los tipos de automedicación más frecuentes y ser sugeridos por el mismo paciente en $49,13 \%$; por familiares en $21,74 \%$, por el técnico de farmacia $14,35 \%$; por vecino o amigo $10,87 \%$ ) por el químico farmacéutico en $3,91 \%$.

En relación a los lugares a los cuales recurren a menudo los estudiantes para adquirir los medicamentos que consumen, se encontró la farmacia o la botica $(91,71 \%)$ seguido por el botiquín familiar $(44,92 \%)$, y en menor prevalencia recurren a los centros de salud (24\%), comparando con Morillo et al ${ }^{(19)}$, los individuos encuestados en su estudio adquieren medicamentos en la farmacia o botica, le sigue el consumo a partir de algunos que le son obsequiados y la compra en venta popular.

En concordancia con los estudios analizados anteriormente la prescripción médica anterior, la influencia de familiares, vecinos y amigos, así como la falta de tiempo y la insuficiencia de los servicios de salud mostraron la prevalencia en los factores que inducen a la automedicación, por tal motivo es importante realizar educación en salud que permita una orientación sobre la automedicación responsable para concientizar a los jóvenes sobre en qué momentos es aconsejable asistir al médico y las consecuencias del consumo indiscriminado y no supervisado de medicamentos de venta libre.

\section{CONCLUSIONES}

Concluimos que en los estudiantes de la Universidad del Magdalena, existe una alta frecuencia de automedicación, siendo predominante en el sexo masculino, en estudiantes entre los 16 y 19 años, ubicados en gran parte en los primeros 4 semestres de las diferentes carreras principalmente en las facultades de Ingeniería, Salud, y Empresariales y en menor proporción Ciencias básicas y Educación; se relaciona esta práctica con los signos y sintomas más frecuentes entre los que se destacan el dolor, sintomas gripales, y gastritis tratados estos con analgésicos, antigripales y antibióticos.

Los principales motivos por los cuales los estudiantes encuestados se automedican se encuentran la levedad de los síntomas seguido por el fácil acceso y comodidad en un continuado por el no gusto a ir al médico. Las fuentes de información de los estudiantes para esta práctica de la automedicación guardan relación con su entorno siendo la recomendación de un familiar cercano, vecino o amigo; a la vez son 
influenciados por la publicidad a través de la televisión y el internet. En gran proporción acuden anualmente al médico o solo cuando se sienten enfermos, dado que consideran ineficientes los servicios de salud, por la falta de tiempo, por falta de dinero, o por desconfianza en el profesional médico. Finalmente, los encuestados manifiestan conocer las consecuencias de esta práctica.

Si bien estos resultados obtenidos muestran la realidad en el campus Universitario, es importante de acuerdo con el perfil de automedicación de los estudiantes de la Universidad del Magdalena realizar acciones de enfermería encaminadas a la educación de la comunidad estudiantil. Así mismo aprovechar la semana de la salud para infundir la automedicación responsable en todas las personas de la Universidad del Magdalena por medio de charlas didácticas, juegos y dinámicas en toda el alma mater donde se trabaje de forma mancomunada bienestar universitario, facultad de salud y el programa de enfermería.

\section{REFERENCIAS}

1. Ibáñez L. Enfermería un viaje a través del cuidado humano. Colombia Editorial Uis, 2013

2. Vera O, Martin J, Ayala E, et al. La automedicación en los estudiantes de la Universidad Nacional de la Región de Lambayeque durante el periodo 2010 2012.

3. French H. Automedicación y Uso Racional del Medicamento en el IPSS. Boletín Terapéutico Toxicológico. № 4. Perú. 1997.

4. De pablo M. La automedicación en la Cultura Universitaria. Revista de Investigación, [Internet]. 2011; 73(35)219-240. [Consultado 12 de enero de 2019]. Disponible http://revistas.upel.edu.ve/index.php/revinvest/article/view/3397/1656

5. Castronuovo C, Chinclana F, Giosso L, Pensa G, Prario M, Rebollo V, Bustos N, Talevi A, Muños s. Automedicacion en estudiantes de la Universidad Nacional de la Plata. Lat. Am. J. Pharm; [Internet]. 2007;26(6) 937 - 944. [Consultado 12 de enero de 2019]. Disponible en: http://www.latamjpharm.org/trabajos/26/6/LAJOP $26 \quad 6 \quad 22$ 0AKQ7SJXN3.pdf

6. Almeida M. Prevalencia y prácticas de automedicación en los estudiantes de primero a quinto año de la Facultad de Medicina de la Universidad San Francisco de Quito en octubre del 2009. [En internet] Ciudad de Quito. Universidad San Francisco de Quito. 2009. [citado el 20 de julio 2020]. Disponible en http://repositorio.usfq.edu.ec/handle/23000/763

7. Paredes Nivia Pinos, Miasso Adriana Inocenti, Tirapelli Carlos Renato. Consumo de benzodiacepinas sin prescripción médica en los/as estudiantes de primer año de la escuela de enfermería de la Universidad de Guayaquil, Ecuador. Rev. Latino-Am. Enfermagem [Internet]. 2008 Aug [citado 2020 July 20] ; 16( spe ): 634-639.

Disponible

en:

http://www.scielo.br/scielo.php?script=sci arttext\&pid=S0104-

11692008000700021\&lng=en. http://dx.doi.org/10.1590/S010411692008000700021.

8. Huancahuari M, Relación entre percepción y la práctica de automedicación responsable en los estudiantes del quinto año de medicina humana de la UNMSM en el mes de enero del 2014. [Internet]. Lima. Universidad Nacional Mayor de San Marcos 2014. [citado 2020 Julio 20] Disponible en: 
https://cybertesis.unmsm.edu.pe/bitstream/handle/20.500.12672/3531/Huancah uari.tm.pdf?sequence $=1 \&$ isAllowed $=y$

9. Vera O., Urcia J., Ayala E., Falla B., \& Díaz C.. La Automedicación en los estudiantes de la Universidad Nacional de la Región Lambayeque durante el periodo noviembre 2010 - Diciembre 2012. Revista Del Cuerpo Médico Del HNAAA [Internet]. 2019 [citado 2020 Julio 20] 9(1), 20 - 31. Disponible en http://www.cmhnaaa.org.pe/ojs/index.php/rcmhnaaa/article/view/147

10. Alba A, Papaqui S, Castillo F, Medina, J, Papaqui J, Ordiano E. Principales causas de por que los estudiantes del área de la salud se automedican. Revista Mexicana De Enfermería Cardiológica. [Internet]. 2019 [citado 2020 Julio 20] 26 (Suplemento): 10. Disponible en: http://ciberindex.com/c/rmec/e26s03

11. Jácome. Roa A. Historia de los medicamentos. [Internet]. 2008. [consultado el 10 de agosto de 2019]

12. Flores S, Santos I. Automedicación en estudiantes de las facultades de Farmacia, Odontología y Enfermería -UNAP- Iquitos 2016. [Internet]. Iquitos. Universidad Nacional De la Amazonia Peruana 2016. [citado 6 Junio 2019] Disponible en: http://repositorio.unapiquitos.edu.pe/bitstream/handle/UNAP/4908/Susan Tesis

Titulo 2016.pdf?sequence=1\&isAllowed=y

13. Asociación médica mundial. Declaración de Helsinki de la AMM - Principios éticos para las investigaciones médicas en seres humanos. 1964.

14. Ministerio de Salud de Colombia. Resolución 8430 de 1993.1993 p. 12 Colombia. C de. Ley 911 de 2004. Código deontológico de Enfermería. 2004 p. 15.

15. López-Cabra C.A, Gálvez-Bermúdez J.M., Domínguez-Domínguez C., UrbinaBonilla A.P., Calderón-Ospina C.A., Automedicación en estudiantes colombianos de pregrado de medicina de la Universidad del Rosario en Bogotá D. C., Colombia. Rev. Colomb. Cienc. Quím. Farm.[internet] 2016 [citado el 2 de junio 2019], vol $45 \quad$ (3): 374-384. Disponible en: https://revistas.unal.edu.co/index.php/rccquifa/article/view/62018/59438

16. Guillem P, Francès F, Gimenez F, Y Sáiz C. Estudio sobre Automedicación en Población Universitaria Española. Rev Clin Med Fam [internet]. 2010 [citado el 6 de junio 2019]. vol.3(2) pp.99-103. Disponible en: http://scielo.isciii.es/scielo.php?script=sci arttext\&pid=S1699695X2010000200008

17. Moraga $\mathrm{C}$, Linares $\mathrm{G}$. Factores asociados a la prevalencia de la automedicación en los estudiantes USFX de Chuquisaca en la ciudad de Sucre. Archivos Bolivianos de Medicina [internet] 2013 20: 88 [citado el 4 de febrero de 2020]. Disponible en: http://www.revistasbolivianas.org.bo/pdf/abm/v20n88/v20n88 a04.pdf

18. Souza L, Silva C, Ferraz G, Faleiros F, Pereira L. Prevalencia y caracterización de la práctica de automedicación para alivio del dolor entre estudiantes universitarios de enfermería. Rev. Latino-Am. Enfermagem [Internet]. 2011 [acceso: 04 de junio 2019] 19(2). Disponible en: http://www.scielo.br/pdf/rlae/v19n2/es 04.pdf

19. Morillo M, Valencia V. Factores relacionados con la automedicación en los estudiantes de enfermería de la Facultad de Ciencias de la Salud de la Universidad Técnica del Norte, en la ciudad de Ibarra en el periodo enero-agosto 2010. 2011. [Citado el 05 de junio 2019]. Disponible en: http://repositorio.ut,n.edu.ec/bi[En internet] tstream/123456789/696/1/06\%20ENF\%20427\%20ARTÍCULO\%20CIENTÍFICO.p df 
20. Gama A, Secoli S. Automedicação em estudantes de enfermagem do Estado do Amazonas - Brasil. Rev. Gaúcha Enferm. [Internet]. 2017 [cited 2020 July 20] ; 38( 1 ): e65111. Disponible en: http://www.scielo.br/scielo.php?script=sci arttext\&pid=S198314472017000100416\&lng=en.http://dx.doi.org/10.1590/19831447.2017.01.65111.

21. Lo Presti Ana. Automedicación: una visión desde la experiencia humana en el contexto comunitario. representaciones sociales. [Posgrado]. Universidad de Carabobo. [internet] Valencia. 2018. Disponible en: http://www.riuc.bc.uc.edu.ve/bitstream/123456789/5904/1/alopresti.pdf

22. Ayala Nicolás et al. Patrones de automedicación y factores influyentes en estudiantes de medicina. Discover Medicine, [En internet] 2020 [citado el 20 de julio 2020] [S.I.], v. 4, n. 1, p. 21-30 ISSN 2521-5612. Disponible en: $<$ https://www.revdiscovermedicine.com/index.php/inicio/article/view/211

23. Altamirano Valeria et al. Automedicación en estudiantes de una residencia universitaria en Chillán, Chile. Revista Cubana de Salud Pública. [En internet] 2019, [citado el 20 de julio 2020] v. 45, n. 1, e1189. Disponible en: https://www.scielosp.org/article/rcsp/2019.v45n1/e1189/es/\#ModalArticles

24. Galarza R. Actitudes y practicas de la automedicación en Estudiantes de Enfermeria de la Universidad Nacional Hermilio Valdizan, Huanuco 2017.: http://200.37.135.58/bitstream/handle/123456789/2327/GALARZA\%20SILVA[En internet] Ciudad de Huanuco. Universidad Nacional Hermilio Valdizan. Huanuco 2017. [citado el 20 de julio 2020]. Disponible en $\% 2 c \% 20$ Rousseau $\% 20$ Paul.pdf? sequence $=1$ \&isAllowed $=y$

25. Garrigoza S, Fernández C, Valdonino M, Bernal B, y Ojeda M. Automedicación en jóvenes de Santa Rosa del Aguaray, Paraguay. Medicina Clínica y Social. [internet] 2017 [citado el 2 de junio 2019], vol 1(2):114-118. Disponible en: https://revistas.unal.edu.co/index.php/rccquifa/article/view/62018/59438

26. Bonilla Y. La Automedicación en los estudiantes de la Universidad Nacional Pedro Ruiz Gallo, Lambayeque. [Pregrado]. Universidad Nacional Pedro Ruiz Gallo; 2017

27. Alam. N; Saffoon N; Uddin R. Self-medication among medical and pharmacy students in Bangladesh. BMC Res Notes; [internet]. 2015; [citado el 07 de julio de 2019]. 8:763 .Disponible en: https://link-springercom.biblioteca.unimagdalena.edu.co/content/pdf/10.1186\%2Fs13104-015-1737$0 . \mathrm{pdf}$

28. Tobón F. Estudio sobre automedicación en la Universidad de Antioquia, Medellín, Colombia. IATREIA [internet]. 2002. VOL. 15 (4) [citado el 5 de agosto de 2019]. disponible en: http://www.scielo.org.co/pdf/iat/v15n4/v15n4a3.pdf

29. Cecilia M, García J, y Atucha N. La automedicación en estudiantes del Grado en Farmacia Self-medication in Pharmacy students. Educ. Mec. [Internet] 2018 [citado el 02 de julio 2019], 19 (5): 277-282. Disponible en: https://reader.elsevier.com/reader/sd/pii/S1575181317301432?token=F50F089F C1FF47B62D7A9AC33FB340F06ECEAB75999E8C496C218A5947749945A8835 4C9635A58E9CC0B34C24DCDFB9E3

30. Felipe D, Ticlla M, Chero V. Automedicación en estudiantes de enfermería en una Universidad Privada en San Juan de Lurigancho. Ágora Rev. Cient. [Internet] 2017; 04(02):e5 [citado el 4 de febrero de 2020]. Disponible en: https://www.researchgate.net/publication/323103222 Automedicacion en estudi antes de enfermeria en una Universidad Privada en San Juan de Luriganc ho 2017 
31. Hermoza-Moquillaza R, Loza-Munarriz C, Rodríguez-Hurtado D, ArellanoSacramento C , Hermoza-Moquillaza V, Automedicación en un distrito de Lima Metropolitana, Perú. Rev Med Hered. [internet] 2016 [citado el 4 de febrero de 2020], 27:15-21.

Disponible en: http://www.scielo.org.pe/pdf/rmh/v27n1/a03v27n1.pdf. 